\title{
Green's Function Technique and Global Optimization in Reconstruction of Elliptic Objects in the Regular Triangle
}

\author{
Antonio Scalia ${ }^{1}$, Mezhlum A. Sumbatyan ${ }^{2}$ \\ ${ }^{1}$ Department of Mathematics and Informatics, University of Catania, Catania, Italy \\ ${ }^{2}$ Faculty of Mathematics, Mechanics and Computer Science, Southern Federal University, \\ Rostov-on-Don, Russia \\ E-mail:scalia@dmi.unict.it; sumbat@math.rsu.ru \\ Received December 28, 2010; revised January 6, 2011; accepted January 9, 2011
}

\begin{abstract}
The reconstruction problem for elliptic voids located in the regular (equilateral) triangle is studied. A known point source is applied to the boundary of the domain, and it is assumed that the input data is obtained from the free-surface input data over a certain finite-length interval of the outer boundary. In the case when the boundary contour of the internal object is unknown, we propose a new algorithm to reconstruct its position and size on the basis of the input data. The key specific character of the proposed method is the construction of a special explicit-form Green's function satisfying the boundary condition over the outer boundary of the triangular domain. Some numerical examples demonstrate good stability of the proposed algorithm.
\end{abstract}

Keywords: Reconstruction, Global Optimization, Green's Function, Triangular Domain, Boundary Integral

\section{Introduction}

In the engineering applications of strength theory the detection and recognition of voids in elastic materials is one of the most important problems of Non-Destructive Evaluation. Various methods are used for this purpose, and one of them is founded on the theory of inverse problems. In order to detect and recognize the image of the void, one may apply over a boundary of the sample a certain type of load, so that to measure the boundary deformation caused by this load. Then one may suppose that the presence (or absence) of interior flaws will influence the measured obtained data. It is also quite natural to suppose that if there is an interior void in the sample then its position and geometry can influence significantly the shape of the deformed boundary. This idea creates a good basis for interior objects reconstruction from the measured data over the boundary of the sample.

A number of theoretical works were devoted to the inverse problems of this kind, with applications to recognition of cracks [1-3]. Some important papers concern uniqueness of the solution, some others develop explicitform analytical results or numerical algorithms [4,5]. Unfortunately, much less results are devoted to reconstruction of volumetric (non-thin) voids in elastic samples under the same conditions and with the same type of input data.

In the present work we study a scalar elastic problem in the domain of a specific form which is the regular (equilateral) triangle. An outer load is applied to its boundary surface, so that the deformation of the domain under this outer force indicates the presence as well as the form of the interior void. We show that so formulated direct problem can be reduced to the Laplace partial differential equation. Then we construct Green's function, which automatically satisfies the trivial boundary condition over the faces of the triangular domain. Such Green's function allows us to formulate the direct problem as a single integral equation holding over the boundary of the void, in the case when a volumetric defect is located inside the elastic triangle. Solution of this integral equation permits to determine the shape of the boundary surface, if the form of the void is known. Further, we formulate the inverse problem, which is to restore the geometry of the void from the measured input data taken as the known deformation of a certain boundary line over some finite-length interval. A specially proposed numerical algorithm is suitable to solve this inverse problem. This is reduced to a sort of global minimization of the discrepancy functional. Finally, we give some examples of application of the proposed method, in the case of the reconstruction (location and geometry) of elliptic voids. 


\section{Mathematical Formulation of the Problem}

Let us consider the regular (equilateral) homogeneous and isotropic elastic triangle under conditions of the (two-dimensional) anti-plane stress-strain state. An elliptic flaw with the boundary $L$ is located in the specimen (see Figure 1). The anti-plane formulation implies that the Cartesian components of the displacement vector $\boldsymbol{u}$ are

$$
\boldsymbol{u}(x, y, z)=\{0,0, w(x, y)\},
$$

where $w$ is the component of the displacement vector in direction $z$. Then the system of equations of equilibrium can be reduced to a single Laplace equation (see, for example, [6])

$$
\begin{gathered}
\mu \Delta \boldsymbol{u}+(\lambda+\mu) \operatorname{grad} \operatorname{div} \boldsymbol{u}=0, \Rightarrow \\
\frac{\partial^{2} w}{\partial x^{2}}+\frac{\partial^{2} w}{\partial y^{2}}=0,
\end{gathered}
$$

where $\lambda$ and $\mu$ are elastic constants. As soon as function $w$ is defined from Equation (2.2), the components of the elastic stress tensor can be found in the following form: $\sigma_{x z}(x, y)=\mu \partial w / \partial x, \sigma_{y z}(x, y)=\mu \partial w / \partial y$. Under condition of the anti-plane problem, the only nontrivial component of the stress vector arising at any elementary area is the tangential stress $T_{z}$ parallel to $z$-axis:

$$
\begin{gathered}
\sigma_{x z}=\mu \frac{\partial w}{\partial x}, \sigma_{y z}=\mu \frac{\partial w}{\partial y}: \\
T_{z}=\sigma_{x z} n_{x}+\sigma_{y z} n_{y} \\
=\mu\left(n_{x} \frac{\partial w}{\partial x}+n_{y} \frac{\partial w}{\partial y}\right)=\mu \frac{\partial w}{\partial n},
\end{gathered}
$$

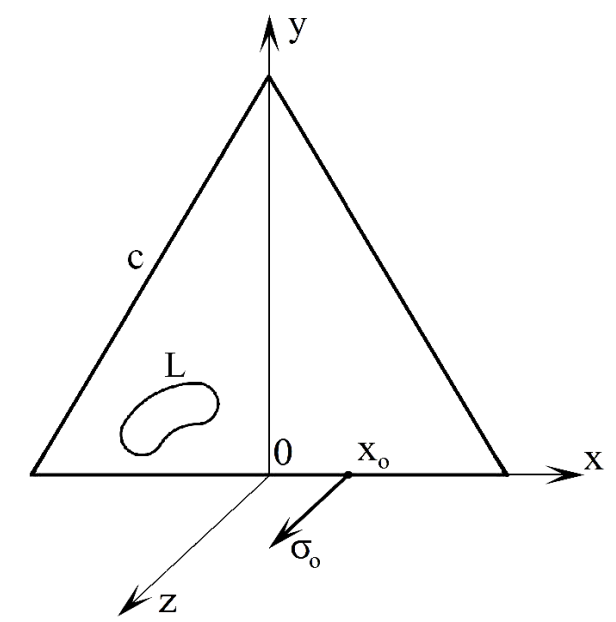

Figure 1. Volumetric flaw in the elastic regular triangle: anti-plane problem. where $\mathbf{n}$ is the unit normal vector to this area. Hence, if we assume that the internal face of the flaw, contour $L$, is free of load, then respective boundary condition is:

$$
\left.\frac{\partial w(x, y)}{\partial n}\right|_{L}=0
$$

Let us assume that a known tangential point force $\sigma_{y x}=\sigma_{0} \delta\left(x-x_{0}\right)$ is applied at the point $\left(x_{0}, 0\right)$ of the boundary line $y=0$ (see Figure 1):

$$
\left.\frac{\partial w(x, y)}{\partial y}\right|_{y=0}=\frac{\sigma_{0}}{\mu} \delta\left(x-x_{0}\right)
$$

With so formulated governing equations the direct problem is to solve Laplace Equation (2.2) with boundary condition (2.4) valid over internal contour $L$. The boundary conditions on the outer surface are given by Equation (2.5) holding over the lower face of the triangle, completed by the Neumann homogeneous boundary conditions analogous to (2.4) on two side faces of the triangle.

The problem is studied in frames of linear elasticity. Therefore, its solution can be represented as a superposition of function $w^{0}(x, y)$ corresponding to outer load (2.5) applied to perfectly continuous (i.e. without any void) triangle, and the one $w^{1}(x, y)$ corresponding to defect located in the triangle whose outer boundary $l$ is free of load, with void $L$ subjected to a certain tangential stress:

$$
w(x, y)=w^{0}(x, y)+w^{1}(x, y) .
$$

This results in the following boundary value problem for function $w^{1}$ :

$$
\begin{aligned}
& \frac{\partial^{2} w^{1}}{\partial x^{2}}+\frac{\partial^{2} w^{1}}{\partial y^{2}}=0, \\
& \left.\frac{\partial w^{1}}{\partial n}\right|_{L}=-\left.\frac{\partial w^{0}}{\partial n}\right|_{L}
\end{aligned}
$$

with the homogeneous Neumann boundary condition on the outer boundary: $\partial w^{1}(x, y) / \partial n=0,(x, y) \in l$.

\section{Green's Function for the Regular Triangle}

In order to reduce the formulated problem to a boundary integral equation (BIE), it is required to construct Green's function in the considered domain. For any single force applied inside the triangle at point $(x, y)$, this Green's function $\Phi(\xi, \eta, x, y)$ should satisfy the Poisson equation

$$
\frac{\partial^{2} \Phi}{\partial \xi^{2}}+\frac{\partial^{2} \Phi}{\partial \eta^{2}}=-\delta(\xi-x) \delta(\eta-y),
$$

$(\xi, \eta)$ inside $l$, 
with the homogeneous free-of-load boundary condition on the triangle's boundary $(\xi, \eta) \in l$ :

$$
\left.\frac{\partial \Phi}{\partial n}\right|_{(\xi, \eta) \in l}=0
$$

where $\mathbf{n}$ is the outer unit normal vector to the boundary $l$.

Following the classical "virtual image" method [8,9], it is clear that for ideal faces of the rectangular domain the homogeneous Neumann boundary conditions (3.2) are automatically satisfied if one arranges a set of delta-function sources symmetrically to the faces, like shown in Figure 2. Then the sought Green's function can be defined as a superposition of 12 infinite series from the following representation valid for all $(\xi, \eta) \in E_{2}$ :

$$
\Phi(\xi, \eta)=\sum_{j=1}^{12} S\left(\xi-x_{j}, \eta-y_{j}, a, b\right), a=\frac{3}{2} c, b=\frac{\sqrt{3}}{2} c,
$$

with

$$
\begin{aligned}
& \left(x_{1}, y_{1}\right)=(x, y) \\
& \left(x_{2}, y_{2}\right)=\left(\frac{3}{4} c-\frac{x}{2}-\frac{\sqrt{3}}{2} y, \frac{\sqrt{3}}{4} c-\frac{\sqrt{3}}{2} x+\frac{y}{2}\right) \\
& \left(x_{3}, y_{3}\right)=\left(\frac{3}{4} c-\frac{x}{2}+\frac{\sqrt{3}}{2} y, \frac{\sqrt{3}}{4} c-\frac{\sqrt{3}}{2} x-\frac{y}{2}\right) \\
& \left(x_{4}, y_{4}\right)=\left(x_{3},-y_{3}\right) \\
& \left(x_{5}, y_{5}\right)=\left(x_{2},-y_{2}\right) \\
& \left(x_{6}, y_{6}\right)=(x,-y) ; \\
& \left(x_{j+6}, y_{j+6}\right)=\left(x_{j}+\frac{3}{2} c, y_{j}+\frac{\sqrt{3}}{2} y\right), j=1, \cdots, 6 .
\end{aligned}
$$

Function $S$ in (3.3) satisfies the Poisson equation in

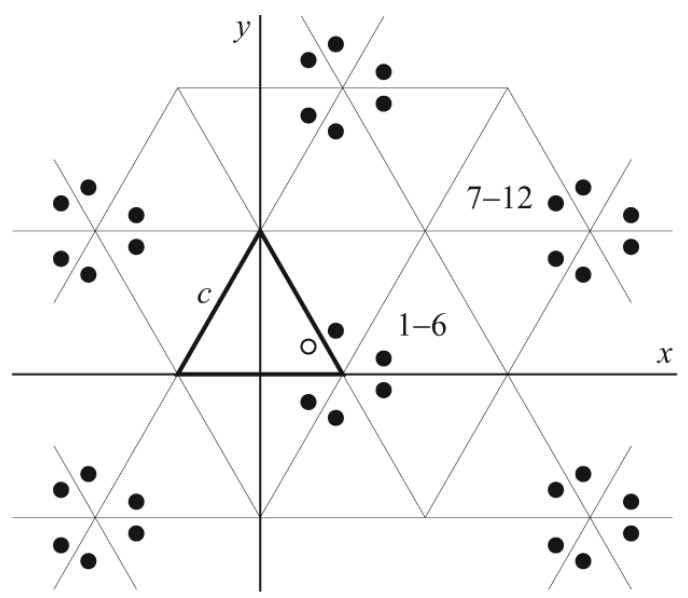

Figure 2. Geometry of virtual images for equilateral triangle. the full $E_{2}$ space:

$$
\begin{aligned}
& \frac{\partial^{2} S(\xi, \eta)}{\partial \xi^{2}}+\frac{\partial^{2} S(\xi, \eta)}{\partial \eta^{2}} \\
& =-\sum_{n, m=-\infty}^{\infty} \delta(2 a n+\xi) \delta(2 b m+\eta), \forall(\xi, \eta) .
\end{aligned}
$$

It is evident that any constant summand added to function $S$ does not change Equation (3.5). This non-uniqueness complicates construction of the sought Green's function. In order to avoid operation with such a singular case, let us introduce a small perturbation replacing Laplace operator in Equation (3.5) by the Helmholtz operator

$$
\begin{aligned}
& \frac{\partial^{2} S_{\varepsilon}(\xi, \eta)}{\partial \xi^{2}}+\frac{\partial^{2} S_{\varepsilon}(\xi, \eta)}{\partial \eta^{2}}+\varepsilon^{2} S_{\varepsilon}(\xi, \eta) \\
& =-\sum_{n, m=-\infty}^{\infty} \delta(2 a n+\xi) \delta(2 b m+\eta),
\end{aligned}
$$

with a certain small wave number $\varepsilon$. Then true solution to Equation (3.5) can be obtained as $S=S_{\varepsilon}, \varepsilon \rightarrow+0$.

Let us apply some classical properties of Dirac's deltafunction:

$$
\begin{gathered}
\delta(\alpha x)=\frac{\delta(x)}{\alpha},(\alpha>0), \\
\sum_{n=-\infty}^{\infty} \delta(n+\beta)=\sum_{n=-\infty}^{\infty} e^{2 \pi n \beta i}=1+2 \sum_{n=1}^{\infty} \cos (2 \pi n \beta) .
\end{gathered}
$$

Then Equation (3.6) can be rewritten as follows

$$
\begin{aligned}
& \frac{\partial^{2} S_{\varepsilon}(\xi, \eta)}{\partial \xi^{2}}+\frac{\partial^{2} S_{\varepsilon}(\xi, \eta)}{\partial \eta^{2}}+\varepsilon^{2} S_{\varepsilon}(\xi, \eta) \\
& =-\sum_{n, m=0}^{\infty} q_{n m} \cos \left(\frac{\pi n \xi}{a}\right) \cos \left(\frac{\pi m \eta}{b}\right), \\
& q_{n m}=\frac{\rho_{n} \rho_{m}}{4 a b} ; \quad \rho_{0}=1, \quad \rho_{n}=2, \quad(n=1,2, \cdots),
\end{aligned}
$$

hence

$$
\begin{aligned}
S_{\varepsilon}(\xi, \eta)= & \sum_{n, m=0}^{\infty}\left(\frac{q_{n m}}{(\pi n / a)^{2}+(\pi m / b)^{2}-\varepsilon^{2}}\right. \\
& \left.\times \cos \left(\frac{\pi n \xi}{a}\right) \cos \left(\frac{\pi m \eta}{b}\right)\right)
\end{aligned}
$$

The last double series can be transformed to a single one if one performs summation over $n$ or $m$. The following tabulated series [10] should be taken into account for this treatment:

$$
\sum_{m=1}^{\infty} \frac{\cos (\pi m z)}{m^{2}+\gamma^{2}}=\frac{1}{2}\left\{\pi \frac{\cosh [\pi \gamma(1-|z|)]}{\gamma \sinh (\pi \gamma)}-\frac{1}{\gamma^{2}}\right\},
$$

therefore, 


$$
\sum_{m=0}^{\infty} \rho_{m} \frac{\cos (\pi m z)}{m^{2}+\gamma^{2}}=\frac{1}{\gamma^{2}}+2 \sum_{m=1}^{\infty} \frac{\cos (\pi m z)}{m^{2}+\gamma^{2}}=\pi \frac{\cosh [\pi \gamma(1-|z|)]}{\gamma \sinh (\pi \gamma)} .
$$

One thus can see from (3.9)-(3.11), with

$$
\begin{gathered}
\gamma=b \sqrt{(\pi n)^{2}-(a \varepsilon)^{2}} /(\pi a), \text { that } \\
S_{\varepsilon}(\xi, \eta)=\sum_{n=0}^{\infty}\left(\frac{\rho_{n} \cos (\pi n \xi / a)}{4 \sqrt{(\pi n)^{2}-(a \varepsilon)^{2}}} \times \frac{\cosh \left[\sqrt{(\pi n)^{2}-(a \varepsilon)^{2}}(b-|\eta|) / a\right]}{\sinh \left[b \sqrt{(\pi n)^{2}-(a \varepsilon)^{2}} / a\right]}\right) . \\
n=0, \text { with asymptotically small } \\
\quad S(\xi, \eta) \sim-(1 / 4 \pi) \ln \left(\xi^{2}+\eta^{2}\right), \\
\xi^{2}+\eta^{2} \rightarrow 0 .
\end{gathered}
$$

The first term here, $n=0$, with asymptotically small $a \varepsilon$ represents itself a certain constant and so, according to what was written above, can be neglected. After all these transformations, with $\varepsilon \rightarrow+0$, the sought Green's function can directly be extracted from (3.12) in the following form:

$$
S(\xi, \eta)=\sum_{n=1}^{\infty} \frac{\cosh [\pi n(b-|\eta|) / a]}{2 \pi n \sinh (\pi n b / a)} \cos (\pi n \xi / a)
$$

It is interesting to control the basic property of any Green's function in the two-dimensional problem: this must possess a logarithmic singularity when $\xi$ and $\eta$ both tend to zero, more precisely one should control that
In order to prove asymptotic relation (3.14), let us take into account the following table series $[10,11]$

$$
\begin{gathered}
\sum_{n=1}^{\infty} \frac{\cos (n z)}{n} e^{-c n}=-\frac{1}{2} \ln \left(1-2 e^{-c} \cos z+e^{-2 c}\right), \\
c \geq 0 .
\end{gathered}
$$

The common term in (3.13) behaves asymptotically as $n \rightarrow \infty$ like in series (3.15) with $z=\pi \xi / a, c=\pi|\eta| / a$. Then the asymptotic behavior of expression (3.13), as $(\xi, \eta) \rightarrow 0$, becomes:

$$
\begin{aligned}
\Phi & \approx \sum_{n=1}^{\infty} \frac{\cos (\pi n \xi / a)}{2 \pi n} e^{-\pi n|\eta| / a}=-\frac{1}{4 \pi} \ln \left(1-2 e^{-\pi|\eta| / a} \cos \frac{\pi \xi}{a}+e^{-2 \pi|\eta| / a}\right) \\
& =-\frac{1}{4 \pi} \ln \left[\left(1-2 e^{-\pi|\eta| / a}\right)^{2}+2 e^{-\pi|\eta| / a}\left(1-\cos \frac{\pi \xi}{a}\right)\right] \approx-\frac{1}{4 \pi} \ln \left(\frac{\pi^{2} \eta^{2}}{a^{2}}+\frac{\pi^{2} \xi^{2}}{a^{2}}\right) \approx-\frac{1}{4 \pi} \ln \left(\xi^{2}+\eta^{2}\right)
\end{aligned}
$$

that is to be proved.

At the end of this section we notice that full structure of the sought Green's function is given by combining Equations (3.3) and (3.13):

$$
\Phi(\xi, \eta, x, y)=\sum_{j=1}^{12} \sum_{n=1}^{\infty} \frac{\cosh \left(\pi n\left(b-\left|\eta-y_{j}\right|\right) / a\right) \cos \left(\pi n\left(\xi-x_{j}\right) / a\right)}{2 \pi n \sinh (\pi n b / a)}
$$

where the set of virtual images is given by Equation (3.4).

\section{BIE for Direct Problem}

Let us come back to the elastic problem shown in Figure 1. We assume that the lower free face is loaded by a known single force at point $\left(x_{0}, 0\right)$, and there is an internal defect with the boundary $L$ inside the triangle. To resolve this problem, one can apply Green's function constructed in the previous section.

One can represent the unknown function $w^{1}(x, y)$ at arbitrary point outside the defect as an integral over its boundary curve $L$, with the use of standard methods of potential theory (see, for example, [12]):

$$
w^{1}(x, y)=\int_{L}\left(w^{1} \frac{\partial \Phi}{\partial n}-\Phi \frac{\partial w^{1}}{\partial n}\right) d l
$$

where both outer unit normal vector $\mathbf{n}(\xi, \eta)$ and elementary arc of length $d l(\xi, \eta)$ are linked to point $(\xi, \eta) \in L$, not to $(x, y)$. It should be noted that for any fixed point $(x, y)$ chosen in the elastic medium the integral in (4.1) should contain additional integration over the outer boundary of the considered elastic domain, contour $l$. However, the second term in such an integrand is trivial due to boundary condition (last relation of Sec- 
tion 2), and the first term vanishes due to the specially constructed Green's function satisfying boundary condition (3.2).

Let us prove that

$$
\int_{L}\left(w^{0} \frac{\partial \Phi}{\partial n}-\Phi \frac{\partial w^{0}}{\partial n}\right) d l=0
$$

for any point $(x, y)$ in the elastic medium. This statement can be proved directly, if one considers Green's integral formula applied to the pair of functions: $w^{0}(\xi, \eta)$ and $\Phi(\xi, \eta, x, y)$ inside contour $L$. Really, both functions are regular in this domain, if point $(x, y)$ is outside $L$, and satisfy there the Laplace equation. Then the application of Green's integral formula immediately re- sults in (4.2) [12].

Now, by summation (4.1) and (4.2), we can express $w^{1}(x, y)$ in terms of boundary values of the full displacement $w(x, y)$ and its normal derivative:

$$
\begin{aligned}
w^{1}(x, y) & =\int_{L}\left(w \frac{\partial \Phi}{\partial n}-\Phi \frac{\partial w}{\partial n}\right) d l \\
& =\int_{L} w(\xi, \eta) \frac{\partial \Phi}{\partial n} d l,
\end{aligned}
$$

due to boundary condition (2.4).

By using the well known limiting value of the potential of double layer [12], if any $(X, Y) \in L$ and contour $L$ is smooth, then

$$
\lim _{(x, y) \rightarrow(X, Y)} \int_{L} w \frac{\partial \Phi}{\partial n}(\xi, \eta, x, y) d l=\frac{w(X, Y)}{2}+\int_{L} w \frac{\partial \Phi}{\partial n}(\xi, \eta, X, Y) d l
$$

With such a limit $(x, y) \rightarrow(X, Y) \in L$, Equation (4.3) allows us to formulate the basic BIE in the form:

$$
\frac{w(X, Y)}{2}-\int_{L} w(\xi, \eta) \frac{\partial \Phi}{\partial n}(\xi, \eta, X, Y) d l=w^{0}(X, Y), \quad(X, Y) \in L
$$

since $w^{1}=w-w^{0}$

For the practical usage of formulas (4.3) and (4.5), it is helpful to write out explicitly the normal derivative of
Green's function. If $\mathbf{n}=\left\{n_{\xi}, n_{\eta}\right\}$ is the outer unit normal vector to the boundary contour $L$ of the defect, then

$$
\begin{aligned}
\frac{\partial \Phi}{\partial n}= & \frac{\partial \Phi}{\partial \xi} n_{\xi}+\frac{\partial \Phi}{\partial \eta} n_{\eta}=\sum_{j=1}^{12}\left[n_{\xi} \frac{\partial S\left(\xi-x_{j}, \eta-y_{j}\right)}{\partial \xi}\right]=-\sum_{j=1}^{12} \sum_{n=1}^{\infty}\left[\frac{n_{\xi} \cosh \left[\pi n\left(b-\left|\eta-y_{j}\right|\right) / a\right] \sin \left[\pi n\left(\xi-x_{j}\right) / a\right]}{2 a \sinh (\pi n b / a)}\right. \\
& \left.+\frac{n_{\eta} \sinh \left[\pi n\left(b-\left|\eta-y_{j}\right|\right) / a\right] \cos \left[\pi n\left(\xi-x_{j}\right) / a\right] \operatorname{sgn}\left(\eta-y_{j}\right)}{2 a \sinh (\pi n b / a)}\right]
\end{aligned}
$$

In order to complete formulation of the basic BIE (4.5) for the direct problem, let us note that its right-hand side, function $w^{0}(X, Y)$ can be obtained similarly to the constructed Green's function. Really, both Green's function and function $w^{0}(X, Y)$ are some solutions for the full triangle caused by a point Dirac's outer applied force. The only difference is that for $w^{0}(X, Y)$ Dirac's delta is applied over the boundary contour, not inside the domain. Therefore, function $w^{0}(X, Y)$ can directly be obtained from representation (3.17) for Green's function, if one sets there point $(x, y)$ approaching the boundary point $\left(x_{0}, 0\right)$, and replaces $(\xi, \eta)$ by $(X, Y)$. Besides, in Equation (3.1) there is sign "minus" in front of Dirac's delta in the right-hand side, hence function $w^{0}(X, Y)$ has to be taken with opposite sign. It should also be noted that with the real source image in Figure 2 tending to the boundary point $\left(x_{0}, 0\right)$, where the outer force is applied, in fact a pair of virtual images (one real and one imaginary) approaches the same point $\left(x_{0}, 0\right)$. For this reason the final resulting limit value should be taken in half. By arranging such a limit, one can obtain the following representation for $w^{0}(X, Y)$ :

$$
w^{0}(X, Y)=-\frac{\sigma_{0}}{4 \pi \mu} \sum_{k=1}^{6} \sum_{n=1}^{\infty}\left(\frac{\cosh \left[\pi n\left(b-\left|Y-y_{k}\right|\right) / a\right]}{n \sinh (\pi n b / a)} \times \cos \left[\pi n\left(X-x_{k}\right) / a\right]\right)
$$


for any $(X, Y)$ inside the triangle. Note that the dimension of the set of virtual images here is in two times less when compared to that in Equation (3.17):

$$
\begin{aligned}
& \left(x_{1}, y_{1}\right)=\left(x_{0}, 0\right) \\
& \left(x_{2}, y_{2}\right)=\left(\frac{3}{4} c-\frac{x_{0}}{2}, \frac{\sqrt{3}}{4} c-\frac{\sqrt{3}}{2} x_{0}\right) \\
& \left(x_{3}, y_{3}\right)=\left(\frac{3}{4} c-\frac{x_{0}}{2},-\frac{\sqrt{3}}{4} c+\frac{\sqrt{3}}{2} x_{0}\right) ; \\
& a=\frac{3}{2} c, \quad b=\frac{\sqrt{3}}{2} c ; \\
& \left(x_{k+3}, y_{k+3}\right)=\left(x_{k}+\frac{3}{2} c, y_{k}+\frac{\sqrt{3}}{2} c\right), k=1, \cdots, 3 .
\end{aligned}
$$

\section{Reconstruction Problem and Some Numerical Results}

If function $w^{0}(x, y),(x, y) \in L$ is determined from
BIE (4.5), the displacement field at arbitrary point of the elastic medium can be calculated by using Equation (4.3):

$$
w(x, y)=w^{0}(x, y)+\int_{L} w(\xi, \eta) \frac{\partial \Phi}{\partial n} d l
$$

where the quantity $\partial \Phi / \partial n$ is given by Equation (4.6). After that, the components of the stress tensor can be calculated as $\sigma_{x z}=\mu \partial w / \partial x, \sigma_{y z}=\mu \partial w / \partial y$. One thus can calculate all physical quantities at arbitrary point $(x, y)$ inside the medium. In particular, the shape of the lower boundary surface $w(x, 0) \in l$ is directly extracted from Equation (5.1). From that representation, we can easily observe the contribution given by the two physically different components:

1) the deformation of the boundary in the perfect (i.e. free of any void) triangle under the applied force $\sigma_{0}$, that is given by the first term in (5.1);

2 ) the contribution given by the influence of presence of the flaw, the second integral term in (5.1).

The latter can be calculated as:

$$
\begin{aligned}
F_{0}(x)= & F_{0}(x, 0)=-\int_{L} w(\xi, \eta) \times \sum_{j=1}^{12} \sum_{n=1}^{\infty} \frac{1}{2 a \sinh (\pi n b / a)} \times\left[n_{\xi} \cosh \left(\pi n \frac{b-\left|\eta-y_{j}\right|}{a}\right) \sin \left(\pi n \frac{\xi-x_{j}}{a}\right)\right. \\
& \left.+n_{\eta} \sinh \left(\pi n \frac{b-\left|\eta-y_{j}\right|}{a}\right) \cos \left(\pi n \frac{\xi-x_{j}}{a}\right) \times \operatorname{sgn}\left(\eta-y_{j}\right)\right] d l,
\end{aligned}
$$

and gives, as has been said above, the contribution to the deformation of the lower boundary surface given by the defect itself.

The inverse reconstruction problem is formulated as follows. Let us assume that a defect of unknown position and shape is located somewhere inside the elastic triangle. Let us apply a concentrated single force $\sigma_{0}$ at a certain point on the lower face $y=0$ and measure the deformation of this lower face. Then, by knowing this measured deformation, it is necessary to predict the position, size, and form of the defect. It is obvious that mathematically the problem is to determine contour $L$ from the known function $F_{0}(x)$. Since another unknown function $\left.w(X, Y)\right|_{(X, Y) \in L}$ is involved in all mathe- matical formulas, this means that mathematically one needs to solve the system of two integral Equations (4.5) and (5.2). This system is nonlinear with respect to any defining equation describing contour $L$. Moreover, since Equation (5.2) is of the first kind, the considered system is ill-posed (see [13]).

The proposed approach is founded on the collocation technique (see, for example, [12]). If contour $L$ is known, then one can arrange a dense set of nodes $\left(\xi_{m}, \eta_{m}\right)$, $m=1, \cdots, N$ belonging to this contour: $\left(\xi_{m}, \eta_{m}\right) \in L, \forall m$, which subdivides it to $N$ small intervals of length $l_{m}$. Then the approximate numerical solution to Equation (4.5) can be obtained by solving the linear algebraic system:

$$
\begin{aligned}
& \sum_{m=1}^{N} a_{i m} w_{m}=w_{i}^{0}, \quad(i=1, \cdots, N), w_{m}=w\left(\xi_{m}, \eta_{m}\right), n_{m}^{\xi}=n_{x}\left(\xi_{m}, \eta_{m}\right), n_{m}^{\eta}=n_{y}\left(\xi_{m}, \eta_{m}\right), \\
& w_{i}^{0}=-\frac{\sigma_{0}}{4 \pi \mu} \sum_{k=1}^{6} \sum_{n=1}^{\infty} \frac{1}{n \sinh (\pi n b / a)} \times \cosh \left[\pi n\left(b-\left|y_{k}\right|\right) / a\right] \cos \left[\pi n\left(x_{0}-x_{k}\right) / a\right],
\end{aligned}
$$

with the elements of matrix $\left\{a_{i m}\right\}$ being given as follows: 


$$
\begin{aligned}
a_{i i}= & \frac{1}{2} ; \text { if } i \neq m: \\
a_{i m}= & -\sum_{j=1}^{12} \sum_{n=1}^{\infty} \frac{l_{m}}{2 a \sinh (\pi n b / a)} \times\left[n_{m}^{\xi} \cosh \left(\pi n \frac{b-\left|\eta_{m}-y_{j}\right|}{a}\right) \sin \left(\pi n \frac{\xi_{m}-x_{j}}{a}\right)\right. \\
& \left.+n_{m}^{\eta} \sinh \left(\pi n \frac{b-\left|\eta_{m}-y_{j}\right|}{a}\right) \cos \left(\pi n \frac{\xi_{m}-x_{j}}{a}\right) \times \operatorname{sgn}\left(\eta_{m}-y_{j}\right)\right] .
\end{aligned}
$$

This system is constructed so that the set of the "inner" discrete integration points $\left\{\left(\xi_{m}, \eta_{m}\right)\right\}$, over which the integration is being performed, coincides with the set of the "outer" nodes $\left\{\left(x=\xi_{i}, y=\eta_{i}\right)\right\}$, which are used to provide the equality between the left- and the right-hand sides in (5.3). This implies that the full set of 12 virtual images is given by expression (3.4) where one should set $x=\xi_{i}, y=\eta_{i}$.

It should also be noted that elements $a_{i m}$ of a long structure are excluded from the diagonal elements (the case $i=m)$. These elements correspond to the case when $(\xi, \eta)=(X, Y)$ in the kernel of integral (4.5). It can easily be proved that these elements remain always bounded for any smooth line $L$, being related to the curvature of the contour at point $(X, Y)$. However, the contribution of such elements to the full sum (5.3) is small (as $\max \left(l_{m}\right) \rightarrow 0$ ) when compared with the con- tribution of the "outer" term in (5.3) outside the integral, which in the discrete form results in the diagonal element $a_{i i}=1 / 2$. Such a treatment allows us to write out the system (5.3) in a shorter form.

When solving the posed reconstruction problem, in practice, the measurements on the deformation of the lower boundary surface cannot be carried out with absolute precision. This predetermines the input data to be known with a certain error. Therefore, the proposed algorithm should provide stability with respect to small perturbations of the input data.

All above developed formulas are valid for arbitrary smooth contour $L$. However, if the flaw is an elliptic cylinder with the semi-axes $A$ and $B$, with its center being located at the point $(c, h)$ and with the angle of inclination $\theta$ respectively axis $x$, then the above formulas can be written in a more concrete form since

$$
\left\{\begin{array}{l}
\xi_{m}=d+\frac{A B \cos \left(\beta_{m}\right)}{\sqrt{A^{2} \sin ^{2}\left(\beta_{m}+\theta\right)+B^{2} \cos ^{2}\left(\beta_{m}+\theta\right)}} \\
\eta_{m}=h+\frac{A B \sin \left(\beta_{m}\right)}{\sqrt{A^{2} \sin ^{2}\left(\beta_{m}+\theta\right)+B^{2} \cos ^{2}\left(\beta_{m}+\theta\right)}} \\
\beta_{m}=\varepsilon(i-0,5) ; \varepsilon=\frac{2 \pi}{N}
\end{array}\right.
$$

Under such conditions the reconstruction problem becomes five-dimensional, because this is to seek five parameters $d, h, A, B, \theta$.

Our approach is founded on an explicit (numerical) resolution of system (5.3) considered as a linear algebraic system for quantities $w_{m}$. Let us represent this system in the operator form

$$
\mathbf{A} w=w^{0}, \mathbf{A}=\left(a_{i m}\right), \quad w=\left(w_{m}\right), w^{0}=\left(w_{i}^{0}\right),(i, m=1, \cdots, N),
$$

then its inversion is

$$
w=\mathbf{A}^{-1} w^{0}, \Rightarrow w_{i}=\left(\mathbf{A}^{-1} w^{0}\right)_{i} .
$$

Obviously, operator $\mathbf{A}^{-1}$ depends on five parameters: $\mathbf{A}^{-1}=\mathbf{A}^{-1}(d, h, A, B, \theta)$, hence the substitution of (5.6) into (5.2) results, in the discrete form, in the overdetermined system of nonlinear equations for parameters $d, h, A, B, \theta$ :

$$
\begin{aligned}
& -\sum_{m=1}^{N} \sum_{j=1}^{12} \sum_{n=1}^{\infty} \frac{1}{2 a \sinh (\pi n b / a)} \times\left[n_{m}^{\xi} \cosh \left(\pi n \frac{b-\left|\eta_{m}-y_{j}\right|}{a}\right) \sin \left(\pi n \frac{\xi_{m}-x_{j}}{a}\right)\right. \\
& \left.+n_{m}^{\eta} \sinh \left(\pi n \frac{b-\left|\eta_{m}-y_{j}\right|}{a}\right) \cos \left(\pi n \frac{\xi_{m}-x_{j}}{a}\right) \times \operatorname{sgn}\left(\eta_{m}-y_{j}\right)\right] \times\left[\mathbf{A}^{-1}(d, h, A, B, \theta) w^{0}\right]_{m} l_{m}=F_{0}\left(x_{q}^{*}\right), \\
& q=1, \cdots, Q, \quad x_{q}^{*} \in(0, a),
\end{aligned}
$$


where $Q$ control points $\left(x_{q}^{*}, 0\right), x_{q}^{*} \in(0, a)$ all belong to the lower face of the triangle. It should be noted that in the case when observation point $(x, y)$ moves to any point $\left(x_{q}^{*}, 0\right)$ belonging to the lower boundary of the triangular domain the number of virtual images $\left(x_{j}, y_{j}\right)$ given by Equation (3.4) reduces again in two times.

Equation (5.7) can be resolved by a minimization of the discrepancy functional [14]:

$$
\begin{aligned}
& \min [\Omega(d, h, A, B, \theta)], \quad \Omega(d, h, A, B, \theta) \\
& =\sum_{q=1}^{Q}\left(\sum _ { m = 1 } ^ { N } \sum _ { j = 1 } ^ { 1 2 } \sum _ { n = 1 } ^ { \infty } \left[n_{m}^{\xi} \cosh \left(\pi n \frac{b-\left|\eta_{m}-y_{j}\right|}{a}\right)\right.\right. \\
& \times \sin \left(\pi n \frac{\xi_{m}-x_{j}}{a}\right)+n_{m}^{\eta} \sinh \left(\pi n \frac{b-\left|\eta_{m}-y_{j}\right|}{a}\right) \\
& \left.\times \cos \left(\pi n \frac{\xi_{m}-x_{j}}{a}\right) \operatorname{sgn}\left(\eta_{m}-y_{j}\right)\right] \\
& \left.\times \frac{\left[\mathbf{A}^{-1}(d, h, A, B, \theta) w^{0}\right]_{m} l_{m}}{2 a \sinh (\pi n b / a)}+F_{0}\left(x_{q}^{*}\right)\right\rangle .
\end{aligned}
$$

It is obvious that in the case of exact input data zero minimal value of $\Omega$ corresponds to exact solution of the inverse reconstruction problem. However, the problem under consideration is nonlinear, hence nobody can guarantee uniqueness of the solution. It should also be noted that, in order to simulate a small error in the input data, we first solve respective direct problem when the shape of the defect is known, and then perturb the obtained solution by a random perturbation. So constructed function $F_{0}$ is used as the approximate input data.

For the minimization of functional $\Omega$ we used in our numerical experiments a version of the random search method [15].

Some examples of the reconstruction are demonstrated in Tables below. For all examples we used $c=1$, $N=50, Q=100, x_{0}=0$.

Here in Table 1 two different flaws located at the same position are considered - a circle and an ellipse directed horizontally, both reconstructions - with exact input data. It is interesting to note that the slope angle for the circle is of no importance in the reconstruction process, and the reconstructed value of $\theta$ plays no role.

Then we studied the stability of the proposed algorithm if the input data is given with an error. As commented above, we add some small perturbation to the solution of respective direct problem. More precisely, each value of the lower face deformation $F_{0}$ is recalculated to a new value by the following formula:

$$
\tilde{F}_{0}=[1+\alpha(2 \zeta-1)] F_{0},
$$

where $\alpha$ is the magnitude of the error (which being
Table 1. Results of the reconstruction with exact input data.

\begin{tabular}{ccccccc}
\hline $\begin{array}{c}\text { Input data } \\
\text { error }\end{array}$ & $d$ & $h$ & $A$ & $B$ & $\theta$ & $\begin{array}{c}\text { Type of } \\
\text { result }\end{array}$ \\
\hline $0 \%$ & 0.000 & 0.300 & 0.150 & 0.150 & 0.000 & Exact \\
& -0.007 & 0.296 & 0.148 & 0.152 & 0.617 & restored \\
$0 \%$ & 0.000 & 0.300 & 0.250 & 0.150 & 0.000 & Exact \\
& 0.009 & 0.308 & 0.253 & 0.157 & -0.002 & restored \\
\hline
\end{tabular}

multiplied by 100 can also be expressed in percents), and $\zeta$ is a random number distributed uniformly over interval $[0,1]$. Some results of such a numerical simulation are shown in Table 2.

It is interesting to notice here that the second example is related to the case when the elliptic flaw is located vertically: in fact, the reconstructed flaw possesses the same property, despite inversion of its principal axes.

Further increase in the error of the input data results in the following table:

Here we notice once again that for the first defect in Table 3 the last reconstructed parameter $\theta$ plays no role, as the flaw is in fact a circle. One thus can admit that this flaw is restored quite well, despite the error in the input data.

From the presented results of the numerical simulation, as well as from many other reconstruction examples performed, we can come to some important conclusions:

1) Generally, precision of the reconstruction is less dependent on the error of the input data than on geometry of the void.

2) The precision of the reconstruction as a rule is good. In some cases almost the same results are obtained with formally different reconstructed geometries. However, with a certain precision, the reconstructed object gives the same original geometry but with another combination of the reconstructed parameters.

3) The worst precision takes place in the reconstruction of prolate ellipses, i.e. with low aspect ratio $B / A$ (see the second example in Table 3 and the second one in Table 2). One can state the following rule natural

Table 2. Results of the reconstruction with relative error $5 \%$ in the input data.

\begin{tabular}{ccccccc}
\hline $\begin{array}{c}\text { Input } \\
\text { data error }\end{array}$ & $d$ & $h$ & $A$ & $B$ & $\theta$ & $\begin{array}{c}\text { Type of } \\
\text { result }\end{array}$ \\
\hline $5 \%$ & 0.200 & 0.150 & 0.100 & 0.030 & $\pi / 4=0.785$ & Exact \\
& 0.205 & 0.144 & 0.102 & 0.036 & 0.797 & restored \\
$5 \%$ & 0.200 & 0.150 & 0.200 & 0.100 & $\pi / 2=1.571$ & Exact \\
& 0.196 & 0.154 & 0.109 & 0.208 & 0.069 & restored \\
\hline
\end{tabular}

Table 3. Results of the reconstruction with relative error $10 \%$ in the input data.

\begin{tabular}{ccccccc}
\hline $\begin{array}{c}\text { Input } \\
\text { data error }\end{array}$ & $d$ & $h$ & $A$ & $B$ & $\theta$ & $\begin{array}{c}\text { Type of } \\
\text { result }\end{array}$ \\
\hline \multirow{2}{*}{$10 \%$} & 0.250 & 0.250 & 0.100 & 0.100 & 0 & Exact \\
& 0.265 & 0.270 & 0.095 & 0.113 & 0.514 & restored \\
$10 \%$ & 0.150 & 0.300 & 0.200 & 0.050 & $-\pi / 4$ & Exact \\
& 0.131 & 0.227 & 0.176 & 0.068 & -0.882 & restored \\
\hline
\end{tabular}


from physical point of view: the more prolate is the void the less is the precision of the reconstruction.

\section{Acknowledgements}

The paper has been supported in part by Italian Ministry of University (M.U.R.S.T.) through its national and local $(60 \%)$ projects. The work is also supported by Russian Foundation for Basic Research (RFBR), Project 10-0100557.

\section{References}

[1] A. Friedman and M. Vogelius, "Determining Cracks by Boundary Measurements," Indiana University Mathematics Journal, Vol. 38, No. 3, 1989, pp. 527-556. doi:10.1512/iumj.1989.38.38025

[2] G. Alessandrini, E. Beretta and S. Vessella, "Determining Linear Cracks by Boundary Measurements: Lipschitz Stability," SIAM Journal on Mathematical Analysis, Vol. 27, No. 2, 1996, pp. 361-375. doi:10.1137/S0036141094265791

[3] A. B. Abda et al., "Line Segment Crack Recovery from Incomplete Boundary Data," Inverse Problems, Vol. 18, No. 4, 2002, pp. 1057-1077. doi:10.1088/0266-5611/18/4/308

[4] S. Andrieux and A. B. Abda, "Identification of Planar Cracks by Complete Overdetermined Data: Inversion Formulae," Inverse Problems, Vol. 12, No. 5, 1996, pp. 553-563. doi:10.1088/0266-5611/12/5/002

[5] T. Bannour, A. B. Abda and M. Jaoua, "A Semi-Explicit
Algorithm for the Reconstruction of 3D Planar Cracks,' Inverse Problems, Vol. 13, No. 4, 1997, pp. 899-917. doi:10.1088/0266-5611/13/4/002

[6] A. S. Saada, "Elasticity: Theory and Applications," 2nd Edition, Krieger, Malabar, Florida, 1993.

[7] N. I. Muskhelishvili, "Some Basic Problems of the Mathematical Theory of Elasticity," Kluwer, Dordrecht, 1975.

[8] R. Courant and D. Hilbert, "Methods of Mathematical Physics," Interscience Publishing, New York, Vol. 1, 1953.

[9] L. Cremer and H. A. Müller, "Principles and Applications of Room Acoustics," Applied Science, London, Vol. 1, 2, 1982.

[10] I. S. Gradshteyn and I. M. Ryzhik, "Table of Integrals, Series, and Products," 5th Edition, Academic Press, New York, 1994.

[11] H. Hardy, "Divergent Series," Oxford University Press, London, 1956.

[12] M. Bonnet, "Boundary Integral Equations Methods for Solids and Fluids," John Wiley, New York, 1999.

[13] A. N. Tikhonov and V. Y. Arsenin, "Solutions of IllPosed Problems," Winston, Washington, 1977.

[14] P. E. Gill, W. Murray and M. H. Wright, "Practical Optimization," Academic Press, London, 1981.

[15] M. Corana et al., "Minimizing Multimodal Functions of Continuous Variables with the Simulated Annealing Algorithm," ACM Transactions on Mathematical Software, Vol. 13, No. 3, 1987, pp. 262-280. doi:10.1145/29380.29864 